\title{
Pós-operatório tardio da substituição do ligamento cruzado cranial no cão
}

\author{
[Late postoperative of cranial cruciate ligament replacement in dog] \\ E.M. Penha ${ }^{1}$, C.M.F. Rezende ${ }^{2 *}$, E.G. Melo ${ }^{2}$, J.V. Doretto ${ }^{1}$, F.A. Araújo ${ }^{3}$, N.T. Vieira ${ }^{1}$ \\ ${ }^{1}$ Aluno de Pós-Graduação - EV-UFMG - Belo Horizonte, MG \\ ${ }^{2}$ Escola de Veterinária - UFMG - Belo Horizonte, MG \\ Caixa Postal 567 \\ 30123-970 - Belo Horizonte, MG \\ ${ }^{3}$ Aluno de graduação - EV-UFMG - Belo Horizonte, MG
}

\begin{abstract}
RESUMO
Realizaram-se avaliações radiográfica e clínica pós-cirúrgica tardia da substituição do ligamento cruzado cranial (LCCr) por fáscia lata autógena em casos de ruptura clínica em 14 cães, de ambos os sexos, com massa corporal média de $28,6 \mathrm{~kg}$ e idade média de 68 meses. As articulações tratadas constituíram o grupo tratado e as contralaterais o grupo-controle, totalizando 28 articulações estudadas. $\mathrm{O}$ tempo médio entre o aparecimento dos sintomas e a cirurgia foi de cinco meses e 15 dias e o decorrido entre a cirurgia e a avaliação pós-cirúrgica, 14 meses. Deambulação normal foi vista em $57,1 \%$ e claudicação discreta em $32,9 \%$ dos casos tratados. Nestes foram observados crepitação, dor, redução da amplitude de movimento articular e do diâmetro da coxa. Ausência de claudicação foi vista em $92,8 \%$ dos casos-controle. Doença articular degenerativa foi verificada radiograficamente nos dois grupos. A técnica empregada para substituição do LCCr foi efetiva clinicamente. Os resultados radiográficos, as vezes, mostravam-se diferente dos clínicos. O resultado foi melhor nos pacientes cuja cirurgia foi realizada após curto período do inicio dos sintomas e o intervalo entre a lesão e o tratamento foi o fator que mais influenciou na recuperação pós-operatória.
\end{abstract}

Palavras-chave: cão, pós-operatório, ligamento cruzado cranial

\begin{abstract}
After the replacement of the cranial cruciate ligament ( $\mathrm{CrCL}$ ) with an autogenous fascia lata graft, radiographic and clinic post-surgical evaluation were performed. Fourteen male and female dogs averaging 68 month-old and $28.6 \mathrm{~kg}$ body weight with unilateral rupture of $\mathrm{CrCL}$ were selected. Twentyeight joints were studied. The treated joints consisted of the treated and non-treated animals. The mean time from the initial onset of the symptoms to the surgery was five months and 15 days and the mean interval between the surgery and the follow-up examination was 14 months. No lameness was observed in $57.1 \%$ of the treated dogs and mild lameness in $42.9 \%$ of them. In this group, articular crepitus, pain, reduced articular motion amplitude and muscular atrophy were observed. No lameness was observed in 98.2\% of the non-treated dogs. These joints showed mild arthrosis. Degenerative joint disease was observed in both groups. The surgical technique was efficient, the dogs showed clinical improvement after surgery, regardless of radiographic signs. The results were better in the dogs submitted to surgery soon after the onset of the symptoms. The interval between the injury and the treatment was the most important factor involved in the recovering after surgery.
\end{abstract}

Keywords: dog, postoperative, cranial cruciate ligament

Recebido em 21 de outubro de 2005

Aceito em 25 de setembro de 2007

*Autor para correspondência (corresponding author)

E-mail: cleuza@ufmg.br

Apoio: FAPEMIG 


\section{INTRODUÇÃO}

A ruptura do ligamento cruzado cranial (LCCr) é uma alteração freqüentemente observada no cão (Coetzee e Lubbe, 1995; Chierichetti et al., 2001) e o retorno funcional do membro é variável (Johnson e Johnson, 1993; Moore e Read, 1995). A doença articular degeneratida (DAD), de caráter progressivo, influencia nos resultados pós-operatórios de substituição do $\mathrm{LCCr}$ (Arnoczky et al., 1979) e seus sinais podem ser observados tanto clínica quanto radiograficamente (Vasseur e Berry, 1992).

A mobilização precoce do membro e a fisioterapia contribuem para minimização dos efeitos da DAD (Muzzi, 2003). Também o uso de condroprotetores como os glicosaminoglicanos, no pós-operatório, é citado como favorável à recuperação clínica do paciente (Arias et al., 2003; Melo et al., 2003).

A avaliação do processo degenerativo é, geralmente, realizada, em veterinária, por meio dos exames clínico e radiográfico. As observações clínicas são direcionadas para avaliação de a estabilidade articular, a hipotrofia muscular, a dor e os padrões de utilização do membro (Arias et al., 2003; Elkins et al., 1991; Muzzi, 2003). Ao exame radiográfico são avaliados a presença e o grau de osteofitose, inclusive na fossa intercondilar (FI) (Vasseur e Berry, 1992; Montgomery et al., 1995; Fitch et al., 1996).

O objetivo deste trabalho foi avaliar os achados clínicos e radiográficos das articulações fêmorotíbio-patelares (AFTP) no pós-operatório tardio, de cães submetidos ao tratamento cirúrgico para substituição do ligamento cruzado cranial pela técnica do auto-enxerto de fáscia lata.

\section{MATERIAL E MÉTODOS}

Entre janeiro de 2000 e dezembro de 2003, após levantamento retrospectivo de casos clínicos de ruptura do LCCr, foram selecionados 14 cães, todos submetidos à substituição do ligamento por auto-enxerto de fáscia lata pela técnica descrita por Schawalder e Gitterle (1989). Desprezaramse os animais com ruptura bilateral e lesões associadas que pudessem interferir na recuperação cirúrgica ou na deambulação do paciente, por exemplo, os portadores de neoplasias articulares, de patologias com manifestação articular, como a babesiose/erliquiose, ou de poliartrites.

Dos 14 animais avaliados, sete eram machos e sete fêmeas com massa corporal média de $28,6 \mathrm{~kg}$, sendo $78 \%$ acima dos $15 \mathrm{~kg}$. Constaram do grupo cinco cães sem raça definida, três Rottweiller; dois Boxer, dois Pitt-bull, um Dobermann e um Poodle. O tempo médio decorrido do início dos sintomas clínicos até o dia da cirurgia foi de cinco meses e 15 dias, e o tempo médio entre a cirurgia e a avaliação póscirúrgica foi de 14 meses.

Foi empregada imobilização pós-operatória do membro com muleta de Thomas modificada durante 14 dias em todos os pacientes. Prescreveram-se para todos cetoprofeno por 10 dias, condroprotetor por 60 dias e fisioterapia. Para a atualização dos dados individuais realizaram-se avaliações clínica e radiográfica das articulações fêmoro-tíbio-patelares direita e esquerda. As articulações tratadas constituíram o grupo de estudo e as contralaterais o de controle. A avaliação dos pacientes ocorreu após tempo mínimo de quatro meses de cirurgia, quando os achados foram transcritos em tabelas. Os parâmetros quantitativos foram classificados e descritos de acordo com a unidade de medida.

Os cães foram submetidos aos exames clínico e radiográfico bilateral das articulações fêmorotíbio-patelares. $\mathrm{O}$ mesmo avaliador realizou todos os testes e durante a interpretação radiográfica as identificações do paciente e do membro estavam protegidas por fita crepe. Utilizou-se apenas contenção manual dos pacientes.

Clinicamente avaliaram-se: deambulação (Tab. 1), a estabilidade articular, diâmetros articular e do terço médio da coxa, crepitação articular (Tab. 2), presença de dor (Tab. 3), amplitudes de flexão e extensão passivas e amplitude de movimentos. Os diâmetros articular e da coxa foram mensurados em centímetros com auxílio de fita métrica; para avaliação das amplitudes de movimentos utilizou-se de um goniômetro de acrílico com círculo completo, e para a estabilidade articular, usou-se $\mathrm{o}$ teste de deslocamento cranial da tíbia em relação ao fêmur, com o membro em extensão e em 


\section{Penha et al.}

semiflexão. De acordo com a instabilidade, classificou-se a articulação em três padrões: firme, discretamente instável e instável. Considerou-se firme quando não se observou deslocamento em teste algum; discretamente instável, quando apresentou mínimo movimento (cerca de $1 \mathrm{~mm}$ ) em qualquer um dos testes; e instável, quando ocorreu deslocamento cranial da tíbia acima da estimativa anterior. Amplitude de movimentos, diâmetros articular e do terço médio da coxa foram mensurados em graus e em centímetros, respectivamente. A deambulação, a crepitação e a dor foram classificadas, individualmente, em graus (Tab. 1, 2 e 3).

Tabela 1. Classificação da deambulação de cães submetidos à substituição do ligamento cruzado cranial, no pós-operatório tardio

\begin{tabular}{ll}
\hline Grau & \multicolumn{1}{c}{ Parâmetro } \\
\hline 1 & $\begin{array}{l}\text { Claudicação ausente; apoio completo do membro com o animal em estação ou durante } \\
\text { atividade física. }\end{array}$ \\
2 & $\begin{array}{l}\text { Claudicação discreta apenas após exercício ou decúbito prolongado. } \\
\text { Claudicação esporádica ao caminhar ou correr, com alívio do peso sobre o membro operado, } \\
\text { inclusive em estação. } \\
\text { Claudicação constante ao caminhar e não apoio do membro ao correr; apoio incompleto em } \\
\text { posição ortostática. } \\
\text { Apoio incompleto ou ausente durante atividades físicas ou em estação. }\end{array}$
\end{tabular}

Tabela 2. Classificação da crepitação articular em cães submetidos à substituição do ligamento cruzado cranial, no pós-operatório tardio

\begin{tabular}{|c|c|}
\hline Grau & Parâmetro \\
\hline 1 & Crepitação ausente \\
\hline 2 & Crepitação discreta: presença de crepitação durante os extremos de flexão e extensão passivas \\
\hline 3 & Crepitação moderada: crepitação intermitente durante a flexão e extensão passivas \\
\hline 4 & $\begin{array}{l}\text { Crepitação acentuada: crepitação intensa e constante durante toda a amplitude de flexão e extensão } \\
\text { passivas. }\end{array}$ \\
\hline
\end{tabular}

Tabela 3. Classificação da dor de cães submetidos à substituição do ligamento cruzado cranial, no pósoperatório tardio

\begin{tabular}{ll}
\hline Grau & \multicolumn{1}{c}{ Parâmetro } \\
1 & $\begin{array}{l}\text { Dor ausente: o animal permite o exercício passivo de todos os movimentos fisiológicos da } \\
\text { articulação sem apresentar relutância ao exame. }\end{array}$ \\
2 & $\begin{array}{l}\text { Dor discreta: o animal permite que a articulação seja manipulada embora apresente sinais de } \\
\text { desconforto articular, voltando o focinho em direção ao avaliador durante o exame. }\end{array}$ \\
3 & $\begin{array}{l}\text { Dor moderada: o animal permite que a aticulação seja manipulada com restrições, e traciona o } \\
\text { membro durante a movimentação articular. }\end{array}$ \\
& $\begin{array}{l}\text { Dor intensa: o animal não permite que a articulação seja manipulada apresentando vocalização e } \\
\text { agressividade. }\end{array}$ \\
\hline Fonte: Adaptado de Muzzi (2003).
\end{tabular}


As articulações operadas (GO) e as controles (GC) foram submetidas à avaliação radiográfica na posição caudocranial. Para o exame radiográfico o animal foi posicionado em decúbito ventral sobre anteparo almofadado, de altura ajustada ao porte do animal, que favorecesse a tração caudal dos membros pélvicos. Sob ambas as articulações fêmorotíbio-patelares colocou-se outro anteparo almofadado. Posicionou-se o tubo emissor de radiação ${ }^{1}$ caudocranialmente em torno de cinco graus com a articulação fêmoro-tíbio-patelar angulada em aproximadamente $105^{\circ}$ e ambas as AFTP rotacionadas internamente em torno de sete graus (Fig. 1 e 2), para visibilização da fossa intercondilar (FI). Para avaliação radiográfica, a FI foi dividida em três áreas (Fig. 3): proximal (a), central (b) e distal (c). Foi utilizada régua milimetrada de acrílico transparente para mensuração do diâmetro das aberturas proximal (a), central (b) e distal (c). Para mensurar a distância entre os epicôndilos (d) traçou-se uma linha paralela ao platô tibial e marcou-se a maior distância entre os epicôndilos femorais. Os índices proximal, central e distal da FI foram obtidos, dividindo-se cada um dos valores, a, b e c, pela distância entre os epicôndilos femorais (d), respectivamente.

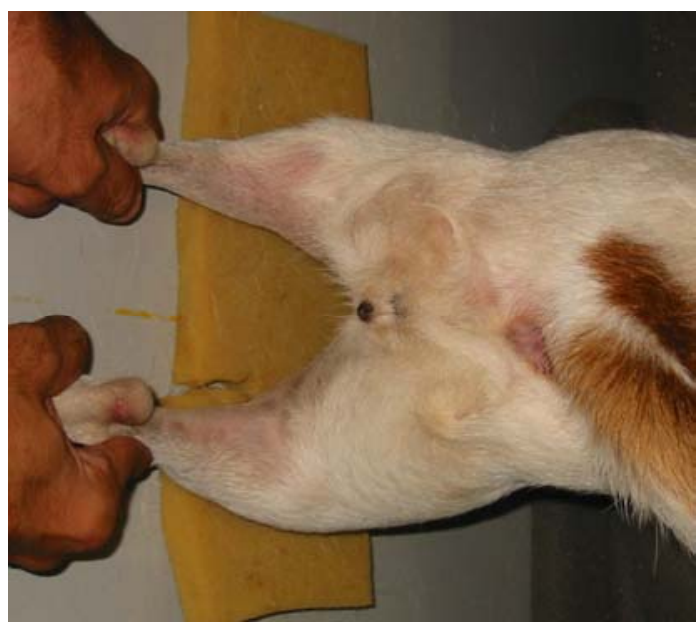

Figura 1. Posicionamento caudocranial para o exame radiográfico da fossa intercondilar no cão. Vista caudal. Os membros pélvicos são tracionados caudalmente e rotacionados internamente em aproximadamente sete graus.

\footnotetext{
${ }^{1}$ Equipamento radiográfico $500 \mathrm{~mA} / 150 \mathrm{Kv}$ (VMI)
}

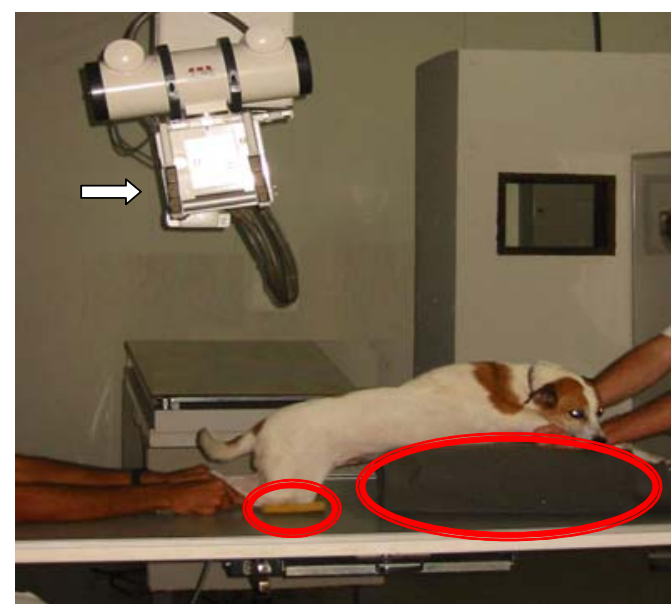

Figura 2. Posicionamento caudocranial para o exame radiográfico da fossa intercondilar no cão. Vista lateral.Ampola emissora de radiação inclinada em aproximadamente cinco graus cranialmente, em relação à fossa intercondilar (seta) e anteparos almofadados sob o torax e sob ambas as articulações femoro-tibio-patelares (eclipses vermelhas).

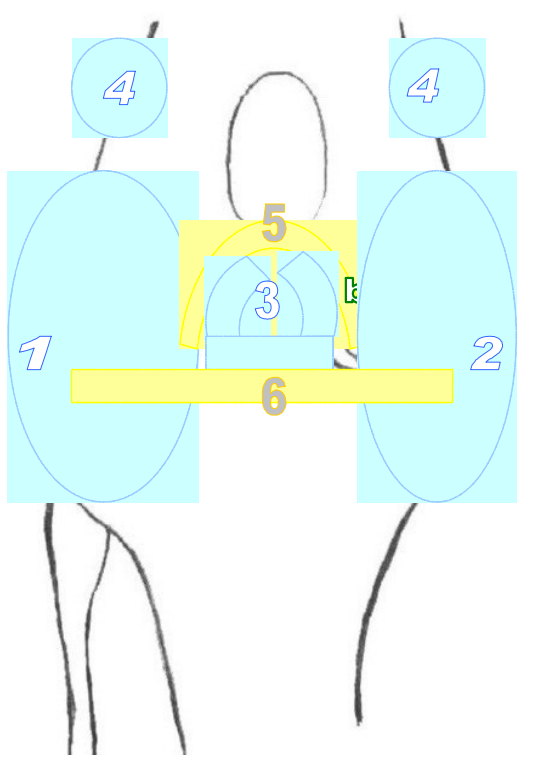

Figura 3. Desenho esquemático da articulação fêmoro-tíbio-patelar com identificação das áreas de observação de osteófitos (1-4), esclerose subcondral (5 e 6) e de medidas das aberturas proximal (a), central(b), distal (c) e entre os epicôndilos femorais (d) para obtenção dos índices da fossa intercondilar de cães submetidos à substituição do ligamento cruzado cranial no pós-operatório tardio. 
Para classificação da DAD foram avaliadas a osteofitose e a esclerose subcondral das AFTP (Tab. 4). Para avaliação da osteofitose, a articulação foi dividida em quatro regiões de observação: (Tab. 4). A esclerose do osso subcondral foi avaliada na fossa intercondilar e no platô tibial. A pontuação das alterações encontra-se na Tab. 4. Para cada articulação foi feito o somatório dos pontos correspondentes às alterações radiográficas encontradas. Segundo o valor assim obtido, a DAD foi descrita como ausente ( 0 ponto), discreta ( 1 a 6 pontos), moderada (7 a 9 pontos) ou grave (10 a 14 pontos).

Tabela 4. Pontuação das alterações radiográficas por região anatômica da articulação femoro-tíbio-patelar de cães submetidos à substituição do ligamento cruzado cranial, no pós-operatório tardio

\begin{tabular}{llcc} 
Área & Região anatômica & Pontuação & Descrição das alterações por região anatômica \\
\hline & $\begin{array}{l}\text { Face lateral: cabeça da } \\
\text { fíbula, superfície epifisária }\end{array}$ & 0 & Ausência de alterações radiográficas.
\end{tabular}
tibial lateral e área epifisária do côndilo femoral lateral.

1 Discreto aumento da radiopacidade com osteofitose em apenas uma das regiões anatômicas.

Discreto aumento da radiopacidade, com discreta osteofitose em

2 mais de uma das regiões anatômicas ou moderado aumento da radiopacidade com acentuada osteofitose em apenas uma das regiões mencionadas.

3 Moderado aumento da radiopacidade, com acentuada osteofitose em mais de uma região.

\begin{tabular}{|c|c|c|c|}
\hline 2 & $\begin{array}{l}\text { Face medial: área epifisária } \\
\text { do côndilo femoral medial e } \\
\text { superfície epifísária tibial } \\
\text { medial }\end{array}$ & $\begin{array}{l}0 \\
1\end{array}$ & $\begin{array}{l}\text { Ausência de alterações radiográficas. } \\
\text { Discreto aumento da radiopacidade com discreta osteofitose em } \\
\text { apenas uma das regiões anatômicas. } \\
\text { Discreto aumento da radiopacidade, com discreta osteofitose em } \\
\text { ambas as regiões anatômicas ou moderado aumento da } \\
\text { radiopacidade com acentuada osteofitose em apenas uma região } \\
\text { anatômica. } \\
\text { Moderado aumento da radiopacidade, com acentuada osteofitose } \\
\text { em ambas as regiões anatômicas. }\end{array}$ \\
\hline 3 & $\begin{array}{l}\text { Espaço intercondilar: fossa } \\
\text { intercondilar (FI) lateral, FI } \\
\text { medial e eminência } \\
\text { intercondilar }\end{array}$ & $\begin{array}{l}0 \\
1\end{array}$ & $\begin{array}{l}\text { Ausência de alterações radiográficas } \\
\text { Discreto aumento da radiopacidade com discreta osteofitose em } \\
\text { apenas uma das regiões anatômicas. } \\
\text { Discreto aumento da radiopacidade, com discreta osteofitose em } \\
\text { mais de uma das regiões anatômicas ou moderado aumento da } \\
\text { radiopacidade com acentuada osteofitose em apenas uma região } \\
\text { anatômica. } \\
\text { Moderado aumento da radiopacidade, com múltiplas áreas de } \\
\text { osteofitose em mais de uma região anatômica. }\end{array}$ \\
\hline 4 & Fabelas medial e lateral & 2 & $\begin{array}{l}\text { Ausência de alterações radiográficas. } \\
\text { Discreto aumento da radiopacidade com discreta osteofitose em } \\
\text { apenas uma das fabelas, sem alterações no formato. } \\
\text { Discreto aumento da radiopacidade, com discreta osteofitose em } \\
\text { ambas fabelas, sem alteração no contorno radiográfico da fabela. } \\
\text { Moderado aumento da radiopacidade, com acentuada osteofitose } \\
\text { em pelo menos uma das fabelas ou perda do contorno } \\
\text { radiográfico. }\end{array}$ \\
\hline 5 & $\begin{array}{l}\text { Osso subcondral na fossa } \\
\text { intercondilar }\end{array}$ & $\begin{array}{l}0 \\
1\end{array}$ & $\begin{array}{l}\text { Esclerose ausente } \\
\text { Esclerose presente }\end{array}$ \\
\hline 6 & $\begin{array}{l}\text { Osso subcondral no platô } \\
\text { tibial }\end{array}$ & $\begin{array}{l}0 \\
1\end{array}$ & $\begin{array}{l}\text { Esclerose ausente } \\
\text { Esclerose presente }\end{array}$ \\
\hline
\end{tabular}


Em cada um dos pacientes foram utilizados dois conjuntos de dados, um inerente ao GO e o outro ao GC. Para a análise estatística foi utilizada a comparação de pares, com os testes Wilcoxon para os dados não paramétricos e $\mathrm{t}$ para os paramétricos. Para tal, foi utilizado o SAEG (Sistema..., 1998).

\section{RESULTADOS E DISCUSSÃO}

Nos testes de estabilidade articular, apenas um (7\%) paciente apresentou instabilidade moderada $(>1,0 \mathrm{~mm})$ no membro operado, o que não foi representativo estatisticamente. Neste caso, verificou-se a ruptura do enxerto, que segundo informação do proprietário, ocorreu cerca de 4-5 semanas após a cirurgia. Nos demais pacientes, observaram-se articulações estáveis (Tab. 5).

Em 11 cães (78,5\%) não foi observada dor e em três $(21 \%)$, dor em graus diferentes, sem diferença significativa (Tab. 5). Entre estes, um com ruptura do enxerto e conseqüente instabilidade apresentou dor moderada. Este cão, de $41 \mathrm{~kg}$, de temperamento indócil, mostrou também apoio insatisfatório do membro, com claudicação moderada. Nesse caso, as avaliações foram feitas antes do tempo previsto, isto é, aos dois meses pós-cirurgia devido à ruptura do enxerto.

Tabela 5. Médias da estabilidade e da amplitude de movimentos da articulação fêmoro-tíbio-patelar e dos parâmetros de deambulação, crepitação articular e dor de cães submetidos à substituição do ligamento cruzado cranial, no pós-operatório tardio

\begin{tabular}{cccccccccc} 
& DCTE & DCTSF & CT & Flexão & Extensão & Amplimov & Deamb & Crepitação & Dor \\
\hline GO & 1,07 & 1,14 & 1,07 & $39,64^{*}$ & $133,79^{*}$ & $94,42^{*}$ & $1,5^{*}$ & $1,64^{*}$ & 1,28 \\
GC & 1,00 & 1,00 & 1,00 & $34,93^{*}$ & $138,71^{*}$ & $104,5^{*}$ & $1,00^{*}$ & $1,07^{*}$ & 1,07 \\
\hline
\end{tabular}

GO: grupo operado; GC: grupo-controle; DCTE: deslocamento cranial da tíbia em relação ao fêmur com o membro em extensão; DCTSF: deslocamento cranial da tíbia em relação ao fêmur com o membro em semiflexão; CT: teste de compressão tibial; amplimov: amplitude de movimentos; deamb: deambulação; crepitação: crepitação articular. Médias seguidas de *, na mesma coluna, diferem entre si $(\mathrm{P}<0,05)$, pelo teste Wilcoxon.

Os outros dois casos crônicos, com menor massa corporal $(23,6$ e 4,9kg) mostravam dor discreta à manipulação. Nesses, a avaliação pós-operatória ocorreu aos 11 meses após a intervenção. Alterações radiográficas foram classificadas como grave (11 pontos) no cão de $23,6 \mathrm{~kg}$, e como discreta, no de $4,9 \mathrm{~kg}$. Estes achados sugerem a contribuição da DAD nas manifestações de dor, ainda que discreta. A menor massa corporal e as alterações radiográficas discretas não foram suficientes para prevenir a expressão clínica da dor.

Uma cadela, de 87 meses, portanto mais velha que os pacientes citados, com sinais radiográficos de DAD discreta, não apresentou dor à manipulação. Nessa paciente, o tempo decorrido do início dos sintomas ao tratamento foi de três meses e o intervalo entre tratamento e avaliação, de cinco.
Apesar da idade, que poderia implicar em DAD mais acentuada, os achados radiográficos e a amplitude de movimentos foram semelhantes e, mesmo assim, a dor não foi detectada segundo os parâmetros de avaliação adotados neste trabalho.

Observou-se entre os grupos diferença entre os valores médios de crepitação e de deambulação (Tab. 6), apesar da ausência de dor na maioria dos animais (78,9\%). Sabe-se que dor articular interfere diretamente na deambulação e pode ter relação com a crepitação (Johnson et al., 1997). Ausência de crepitação foi verificada em sete cães (50\%), crepitação moderada em duas (14,3\%) articulações tratadas e crepitação discreta em cinco $(35,7 \%)$, sendo também observado no grupo-controle um $(7,1 \%)$ caso de crepitação discreta.

Tabela 6. Médias e desvio-padrão dos diâmetros $(\mathrm{cm})$ articular e do terço médio da região da coxa de cães submetidos à substituição do ligamento cruzado cranial, no pós-operatório tardio

\begin{tabular}{lccccc}
\hline & Crepitação & Deambulação & Dor & DArtic & DTM \\
\hline GO & $1,64 \pm 0,74^{*}$ & $1,5 \pm 0,65^{*}$ & $0,28 \pm 0,61$ & $26,53 \pm 6,72^{*}$ & $33,92 \pm 10,23^{*}$ \\
GC & $1,07 \pm 0,27^{*}$ & $1,00 \pm 0,0^{*}$ & $1,07 * 0,27$ & $25,92 \pm 6,45^{*}$ & $35,64 \pm 10,80^{*}$ \\
\hline
\end{tabular}

GO: grupo operado; GC: grupo-controle; DArtic: diâmetro articular; DTM: diâmetro do terço médio da região da coxa.

Médias seguidas de *, na mesma coluna, diferem entre si pelo teste $\mathrm{t}(\mathrm{P}<0,05)$. 
Deambulação normal foi verificada em 13 $(92,8 \%)$ cães do $\mathrm{GC}$ e em oito $(57,1 \%)$ do GO. Claudicação discreta foi observada em um $(7,2 \%)$ cão do grupo-controle e em seis $(42,9 \%)$ do grupo operado, sendo que em um $(7,1 \%)$ desses a claudicação era esporádica, com alívio de peso no membro em estação. Não foi possível, entretanto, nos moldes deste trabalho, relacionar o tempo pós-cirúrgico mínimo ao retorno funcional do membro. Acredita-se, porém, que de maneira similar à observada em trabalhos experimentais (Arias et al., 2003; Melo et al., 2003; Muzzi, 2003, Biasi et al., 2005) tenha ocorrido melhora progressiva da claudicação e do apoio durante o pós-operatório imediato. $\mathrm{O}$ tempo médio para o retorno funcional do membro operado varia em torno de quatro meses (Vasseur et al., 1985; Elkins, 1991; Moore e Read, 1995) podendo se prolongar quando da presença de DAD (Arnoczky et al., 1979), inclusive com apoio insatisfatório contínuo do membro operado, mesmo após anos de observação (Moore e Read, 1995).

Convém salientar que os animais deste trabalho são de casuística clínica, com idade média de $68,4 \pm 24,2$ meses. A observação de crepitação articular dentre os oito $(57,1 \%)$ animais do GO que apresentaram deambulação normal confirma o relato de que crepitação articular pode estar presente mesmo na ausência de dor, conforme citado por Coetzee e Lubbe (1995). Apesar de a crepitação ser observada na presença de instabilidade articular (Chierichetti et al., 2001), o envelhecimento é um dos fatores descritos como predisponentes (Vasseur et al., 1985; Johnson e Johnson, 1993). Acredita-se que a crepitação nos pacientes deste trabalho seja devido à formação óssea periarticular decorrente da DAD, como já citado por Muzzi (2003).

Outro fator que provavelmente agrava a degeneração articular é o tempo entre a ruptura do ligamento e a cirurgia. Neste trabalho foi de cinco meses e meio, média acima da citada na literatura (Johnson e Johnson, 1993; Johnson et al., 1997; Chierichetti et al., 2001). Não foi possível relacionar, entretanto, os sinais de DAD ao tempo decorrido entre o início dos sintomas e a cirurgia, nem a sua progressão desde a cirurgia até o momento de avaliação. Apesar de apenas $57,1 \%$ dos pacientes recuperarem a deambulação normal, sem claudicação, é de se esperar, que a intervenção cirúrgica proporcione algum benefício quer seja na promoção de conforto, quer seja na redução da velocidade de progressão da DAD. Acredita-se que com avaliações seriadas seja possível relacionar o tempo póscirúrgico ao retorno funcional do membro operado, bem como avaliar a influência da DAD na recuperação clínica pós-operatória.

Outros fatores, além da dor, estão envolvidos no relato da qualidade da deambulação tais como: a individualidade de cada avaliador, tempo de observação, evolução da DAD, efeito do estresse inerente ao paciente e aquele provocado pelo ambiente estranho.

Na mensuração da flexão, extensão e amplitude de movimentos foram encontrados valores, em média, menores no grupo operado que no controle. Dentre os fatores responsáveis pela limitação da função do membro tem-se, além da dor, a presença da DAD em graus variados quer seja provocada pela injúria cirúrgica, quer seja pela doença em si. Vale lembrar que todas as articulações foram imobilizadas com muleta de Thomas modificada, por duas semanas, e que foi sugerida fisioterapia pós-cirúrgica. Apesar da indicação, apenas cinco $(35,7 \%)$ proprietários disseram ter seguido, pelo menos parcialmente, $o$ recomendado.

Em cinco $(35,7 \%)$ pacientes a amplitude de movimentos da articulação operada não diferiu do controle. Em nove $(64,3 \%)$ verificou-se diferença significativa e em três desses $(21,4 \%)$, cujo tratamento só foi realizado após sete meses do início dos sintomas, a diferença foi maior que o dobro da diferença média entre os grupos. Acredita-se que as alterações periarticulares responsáveis pela maior diminuição da amplitude de movimentos sejam decorrentes da evolução da DAD.

A idade dos pacientes, o tempo de evolução da DAD e a ausência de fisioterapia são fatores que favorecem o surgimento dessas alterações. Muito embora imobilização pós-operatória possa ser incriminada como um dos fatores responsáveis pela redução da amplitude de movimentos (Videman, 1987), acredita-se que o período de imobilização de duas semanas, utilizado neste trabalho, não foi suficiente para que os efeitos nocivos da imobilização prolongada atuassem gravemente na articulação (Muzzi, 2003). 
O diâmetro médio do terço médio da coxa no grupo operado apresentou valores, em média, menores que no grupo-controle (Tab. 6). Na maioria dos casos $(12 ; 85,7 \%)$ a diferença foi significativa. Em dois animais a diferença do diâmetro entre o membro operado e o controle estava associada à redução da amplitude de movimentos. A cirurgia foi realizada aos 24 e aos sete meses após a ruptura e a avaliação pósoperatória aos cinco e aos 60 meses pósintervenção, respectivamente. As alterações radiográficas foram mais evidentes nestes casos. Credita-se a este conjunto de alterações, as conseqüências da DAD.

A hipotrofia muscular por diminuição do uso do membro, após ruptura do ligamento cruzado cranial, é citada com freqüência na literatura (Shires et al., 1984; Vasseur e Berry, 1992; Coetzee e Lubbe, 1995; Arias et al., 2003; Chierichetti et al., 2001). Acredita-se que as características de recuperação pós-operatória observadas neste trabalho estejam relacionadas com o grau de DAD pré-tratamento. Em sete $(50 \%)$ pacientes, verificou-se diferença significativa no diâmetro articular, provavelmente devido à fibrose periarticular.

O primeiro achado radiográfico decorrente da DAD é a efusão articular (Shires et al., 1984). Em três animais (21\%) apresentou-se essa alteração no momento da avaliação pósoperatória. Em associação à efusão, em dois desses pacientes houve sinais radiográficos de DAD moderada (Tab. 4) e no outro, DAD discreta.

Radiograficamente foram observados no grupo operado, osteofitose e esclerose subcondral mais intensas que no grupo-controle. No GO, a DAD manifestou-se com intensidade discreta a moderada e no GC, discreta (Tab. 4). Houve variações individuais que envolviam principalmente tempos diferentes de avaliação pós-cirúrgica e tempo de evolução entre o diagnóstico e o tratamento. Em um mesmo animal, a diferença mínima de pontuação radiográfica entre o membro operado e o controle foi de um ponto e a maior de 11. Essa variação não foi relacionada ao tempo de pósoperatório. Observou-se, entretanto, que nos pacientes em que o período entre o surgimento dos sintomas clínicos e a cirurgia foi maior, a pontuação radiográfica também foi maior.
DAD discreta foi observada em quatro $(28,5 \%)$ articulações do grupo operado (Tab. 7) e em 13 $(92,8 \%)$ do grupo-controle. Observaram-se cinco $(35,7 \%)$ articulações no GO e uma $(7,2 \%)$ articulação controle com alterações moderadas. Nenhuma alteração grave foi verificada nas articulações do $\mathrm{GC}$, ao passo que cinco $(35,7 \%)$ do GO manifestaram DAD avançada ao exame radiográfico. Não houve relação entre dor ou deambulação com os achados radiográficos.

Tabela 7. Médias e desvios-padrão da pontuação radiográfica e expressão numérica média da interpretação qualitativa da DAD em cães submetidos à substituição do ligamento cruzado cranial, no pós-operatório tardio

\begin{tabular}{lcc}
\hline & Pontos & DAD \\
\hline GO & $8,35 \pm 2,5^{*}$ & $2,07 \pm 0,83^{*}$ \\
GC & $3,64 \pm 1,5^{*}$ & $1,07 \pm 0,27^{*}$ \\
\hline
\end{tabular}

GO: grupo operado; GC: grupo-controle; DAD: doença articular degenerativa.

Médias seguidas de *, na mesma coluna, diferem entre si $(\mathrm{P}<0,05)$ pelo teste Wilcoxon.

Os índices da FI não apresentaram diferenças significativas entre os grupos, com valores médios de $0,18,0,24$ e 0,26 para os índices proximal, central e distal, respectivamente (Tab. 8). Fitch et al. (1995) encontraram, em articulações normais, índices de $0,18,0,25$ e 0,32 , respectivamente, para as aberturas cranial, central e distal. No presente trabalho, verificouse que o índice distal médio foi diferente daquele citado por Fitch et al. (1995). Não foram encontradas na literatura referências aos valores a partir dos quais pode-se afirmar que a FI está estreita. Apesar de respeitadas as angulações sugeridas por Fitch et al. (1995), acredita-se que a mudança de decúbito tenha interferido nos valores obtidos para os índices distais.

Tabela 8. Médias dos índices proximal, central e distal da fossa intercondilar de cães submetidos à substituição do ligamento cruzado cranial, no pós-operatório tardio

\begin{tabular}{lccc}
\hline & IFIpr & IFIce & IFIdi \\
\hline GO & $0,18 \pm 0,04$ & $0,24 \pm 0,04$ & $0,26 \pm 0,07$ \\
GC & $0,18 \pm 0,01$ & $0,24 \pm 0,03$ & $0,25 \pm 0,02$ \\
\hline
\end{tabular}

GO: grupo operado; GC: grupo-controle; IFIpr: índice da FI proximal; IFIce: índice da fossa intercondilar central; IFIdi: índice da FI distal.

A mudança no decúbito, entretanto, não impediu a avaliação da FI, uma vez que o índice distal da 
articulação controle foi usado como parâmetro. Em um paciente, no qual o tempo decorrido entre o surgimento dos sintomas clínicos até o tratamento cirúrgico foi de dois anos, observouse preenchimento acentuado da fossa intercondilar. Nesse verificou-se diferença significativa em todos os índices, entre a articulação operada e a controle, a saber: a) cranial- GO 0,05 e GC: 0,18; b) central- GO 0,13 e GC 0,21 ; e c) distal- GO 0,08 e GC 0,24 . O preenchimento da fossa intercondilar, evidenciado pela formação de osteófitos, em decorrência de instabilidade crônica da AFTP e de estabilização articular insatisfatória tem sido relatado na literatura (Fitch et al., 1995; Fitch et al., 1996; Selmi, 2002). O estreitamento da fossa pode também ter origem congênita e o atrito constante do LCCr com o teto da FI predispõe à ruptura (Montogomery et al., 1995). O estreitamento da FI, segundo Fitch et al. (1995), e Montgomery et al. (1995) pode contribuir para a ruptura do enxerto, além de dificultar ou mesmo impossibilitar a substituição do ligamento.

Houve um caso de estreitamento da FI. Neste, a diferença radiográfica entre a AFTP operada e a controle, após oito meses de cirurgia, foi de 11 pontos (Tab. 5). Essa foi maior que a diferença média entre os grupos (4,7 pontos). Em outro cão, a diferença entre as imagens radiográficas das articulações operada e controle, após dois anos de cirurgia, foi de um ponto (Tab. 5). Esse paciente foi operado após um mês do início dos sintomas e avaliado após 23 meses de cirurgia. Isso confirma o benefício do tratamento precoce.

\section{CONCLUSÕES}

As imagens radiográficas não representam os dados clínicos, as avaliações clínica e radiográfica confirmam que o intervalo entre lesão e tratamento influencia a recuperação dos cães submetidos à substituição do $\mathrm{LCCr}$; e a técnica cirúrgica de substituição do $\mathrm{LCCr}$ por auto-enxerto de fáscia lata, apesar de efetiva, não impede a progressão da DAD.

\section{REFERÊNCIAS BIBLIOGRÁFICAS}

ARIAS, S.A.A.; REZENDE, C.M.F.; MELO, E.G. et al. Avaliação radiológica, artroscópia e histológica da membrana sinovial do joelho de cães tratados com associação de sulfato de sódio e hialurônico. Arq. Bras. Med. Vet. Zootec., v.55, p.421-429, 2003.

ARNOCZKY, S.P.; TARVIN, G.B.; MARSHALL, J.L. et al. The over-the-top procedure: A technique for anterior cruciate ligament substitution in the dog. J. Am. Anim. Hosp. Assoc., v.15, p.283-290, 1979.

BIASI, F.; RAHAL, S.C.; VOLPI, R.S. et al. Reconstrução do ligamento cruzado cranial em cães, associado ou não ao sulfato de condroitina. Arq. Bras. Med. Vet. Zootec., v.57, p. 442-447, 2005.

CHIERICHETTI, A.L.; ALVARENGA, J.; PEDRO, C.R. et al. Ruptura do ligamento cruzado cranial. Estudo comparativo da técnica extra-articular com enxerto autógeno de fáscia lata com e sem artrotomia exploratória. Clín. Vet., v.4, p.34-42, 2001.

COETZEE, G.L.; LUBBE, A.M. A prospective study comparing two fascial reconstruction techniques to stabilize the cranial cruciate deficient stifle in the dog. Vet. Comp. Orthop. Traumatol., v.8, p.82-90, 1995.

ELKINS, D.A.; PECHMAN, R.; KEARNEY, M.T. et al. A retrospective study evaluating the degree of degenerative joint disease in the stifle joint of dogs following surgical repair of anterior cruciate ligament rupture. J. Am. Anim. Hosp. Assoc., v.27, p.533-540, 1991

FITCH, R.B.; HATHCOOCK, J.T.; MONTGOMERY, R.D. et al. Radiographic and computed tomographic evaluation of the canine intercondylar fossa in normal stifles and after notchplasty in stable and unstable stifles. Vet. Radiol. Ultras., v.1, p.266-274, 1996.

FITCH, R.B.; MONGOMERY, R.D.; MILTON, J.L. et al. The intercondylar fossa of the normal canine stifle: An anatomic and radiographic study. Vet. Surg., v.24, p.148-155, 1995.

JOHNSON, J.M.; JOHNSON, A.L. Cranial cruciate ligament rupture. Pathogenesis, diagnosis and postoperative rehabilitation. Vet. Clin. N. Am.; Small Anim. Pract., v.23, p.717733, 1993.

JOHNSON, J.M.; JOHNSON, A.L.; PIJANOWSKI, G.J. et al. Rehabilitation of dogs with surgically treated cranial cruciate ligamentdeficient stifles by use of electrical stimulation of 
muscles. Am. J. Vet. Res., v.58, p.1473-1478, 1997.

MELO, E.G.; REZENDE, C.M.F.; GOMES, M.G. et al. Sulfato de condroitina e hialuronato de sódio no tratamento da doença articular degenerativa em cães. Aspectos clínicos e radiográficos. Arq. Bras. Med. Vet. Zootec., v.55, p.35-43, 2003.

MONTGOMERY, R.D.; FITCH, D.V.; HATHCOOK, J.T. et al. Radiographic imaging on the canine intercondilar fossa. Vet. Radiol. Ultras., v.35, p.276-282, 1995.

MOORE, K.W.; READ, R.A. Cranial cruciate ligament rupture in the $\operatorname{dog}-$ a retrospective study comparing surgical techniques. Aust. Vet. J., v.72, p.281-285, 1995.

MUZZI, L.A.L. Fisioterapia e imobilização temporária na substituição artroscópica do ligamento cruzado cranial em cães (Estudo experimental). 2003. 79f. Tese (Doutorado) Escola de Veterinária, Universidade Federal de Minas Gerais, Belo Horizonte.

SCHAWALDER, P.; GITTERLE, E. Eigene methoden zur operativen rekonstruktion bei rupturen des vorderen und hinteren kreuzbandes. Kleintierpraxis, v.7, p.323-330, 1989.
SELMI, A.L. A incisuroplastia troclear na estabilização articular com retalho de fascia lata, após transecção do ligamento cruzado cranial em cães. 2002. Tese (Doutorado) Faculdade de Medicina Veterinária e Zootecnia, Universidade Estadual Paulista, Jaboticabal, SP.

SHIRES, P.K.; HULSE, D.A.; LIU, W. et al. The under-and-over fascial replacement technique for anterior cruciate ligament rupture in dogs: A retrospective study. J. Am. Anim. Hosp. Assoc., v.20, p.69-77, 1984.

SISTEMA de análise estatísticas e genética SAEG - Versão 8.0. viçosa: UFV, 1998.

VASSEUR, P.B.; BERRY, C.R. Progression of stifle osteoarthrosis following reconstruction on the cranial cruciate ligament in 21 dogs. J. Am. Anim. Hosp. Assoc., v.28, p.129-136, 1992.

VASSEUR, P.B.; POOL, R.R.; ARNOCZKY, S.P. et al. Correlative biomechanical and histologic study of the cranial cruciate ligament in dogs. Am. J. Vet. Res., v.46, p.1842-1854, 1985.

VIDEMAN, T. Connective tissue and immobilization: Key factors in musculoskeletal degeneration? Clin. Orthop. Rel. Res., v.221, p.26-32, 1987. 